\title{
In Search of the Lost Nodes in BANs
}

\section{Costas Michaelides, Maria Iloridou and Foteini-Niovi Pavlidou \\ School of Electrical and Computer Engineering \\ Aristotle University of Thessaloniki}

\begin{tabular}{|l|}
\hline Abstract \\
\hline Communication in Body Area Networks (BANs) involves \\
extremely weak signals due to safety regulations. Mobility adds \\
one more layer of complexity as it has an effect on path loss \\
depending on the activity. In this work, we improve the quality \\
of service (QoS) by searching for the lost nodes. Specifically, \\
an Emergency Phase (EP) is added in the superframe. During \\
EP, each connected node to the hub transmits a rescue beacon \\
to reach distressed nodes, i.e. not connected. When a \\
distressed node receives a rescue beacon, it participates in the \\
corresponding EP. Its data packets are buffered and forwarded \\
to the hub by the connected node. This feature is proposed as \\
an enhancement of IEEE Std 802.15.6-2012. \\
\hline
\end{tabular}

\begin{tabular}{|c|c|c|c|}
\hline \multicolumn{4}{|c|}{ Introduction } \\
\hline \multicolumn{4}{|c|}{$\begin{array}{l}\text { The standard for BANs was released back in } 2012 \text {. It supports } \\
\text { a variety of data rates for the communication of the nodes in } \\
\text { one-hop star or two-hop extended star topology. The nodes can } \\
\text { be on, in or close to the human body. Channel access during } \\
\text { the superframe can be random, improvised or scheduled. } \\
\text { - In this work, we propose the use of a special random access } \\
\text { phase (RAP) during the superframe, called EP, which takes } \\
\text { place after RAP1. We test this feature using a channel } \\
\text { model for } 2.4 \mathrm{GHz} \text { and mobility with real traces from Mocap } \\
\text { Database HDM05. Multi-hopping is restricted to two hops. } \\
\text { The simulation is implemented using Castalia, a framework of } \\
\text { OMNeT++. }\end{array}$} \\
\hline Hub & RAP1 & EP & \\
\hline & & & \\
\hline $\begin{array}{l}\text { Connected } \\
\text { Node }\end{array}$ & RAP1 & EP & \\
\hline & & $\dot{i}$ & \\
\hline $\begin{array}{l}\text { Distressed } \\
\text { Node }\end{array}$ & & EP & \\
\hline
\end{tabular}

\section{MAC}

Our MAC protocol is heavily based on Baseline MAC which was implemented by the creators of Castalia as their baseline for IEEE Std 802.15.6-2012. The proposed EP is an additional RAP with the purpose of reaching the distressed nodes and forwarding their packets. The access method is CSMA/CA using the contention windows of the standard.

- During EP, each connected node to the hub can optionally send a rescue beacon to reach any possible distressed node, i.e. not connected. When a distressed node receives a rescue beacon from a connected node, it participates in its EP. When a connected node receives a data packet, it responds with an I-Ack and buffers the packet to transmit it during the next RAP1. If its buffer is full, the packet is dropped. This takes place in every superframe. When a distressed node approaches the hub, it connects and participates in RAP1.

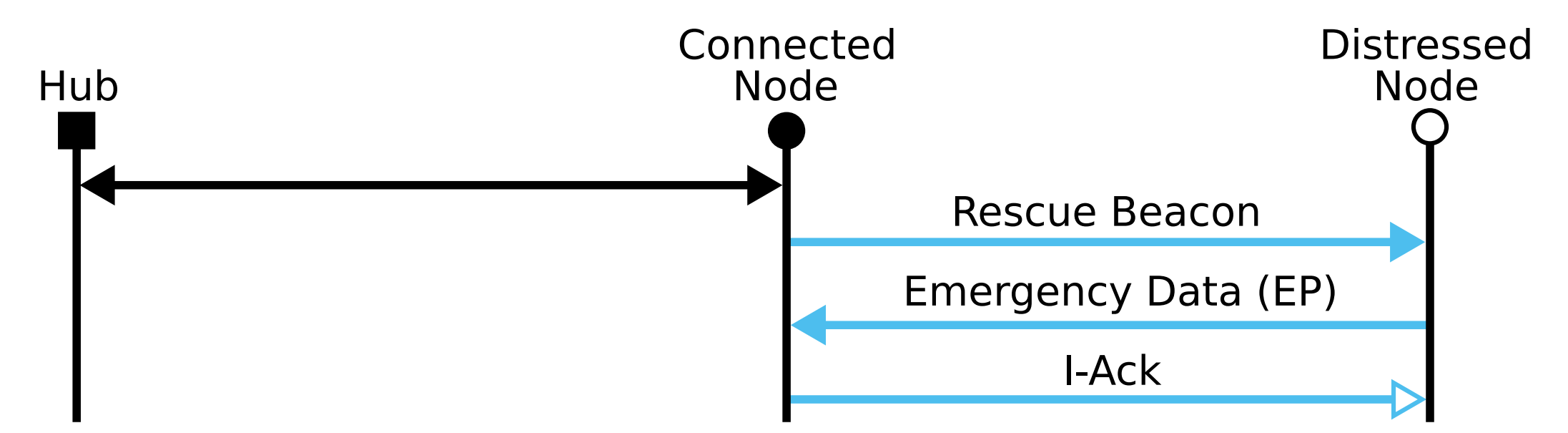

Figure 2: Communication with enabled EP

\section{Mobility model}

After several tests, we realised that the way to implement human mobility accurately is to use empeirical data instead of random mobility models. Trace based mobility is possible because there is a great amount of motion capture data available online.

- Our model uses a dataset of real traces of human body parts for an everyday activity from HDM05. You can watch an animated clip of it on YouTube by scanning the QR code.

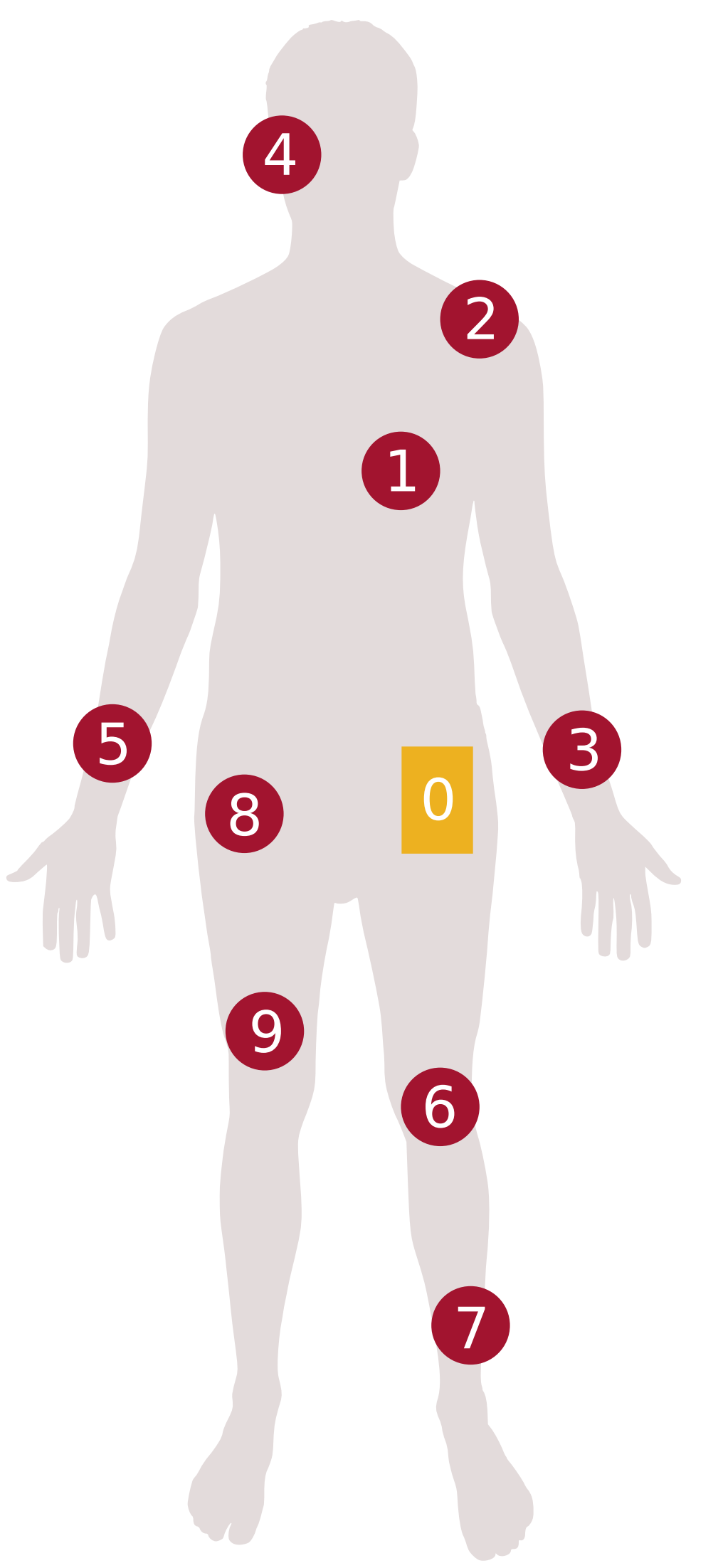

Figure 3: Node placement $\sim$ Simulation

\begin{tabular}{|c|c|}
\hline \multicolumn{2}{|c|}{ Simulation } \\
\hline Parameter & Value \\
\hline Time & $60 \mathrm{~s}$ \\
\hline Nodes & $9+$ Hub \\
\hline Application & Throughput \\
\hline Payload & 100 bytes \\
\hline Packet rate & 20 packets/s \\
\hline TX power & $-10 \mathrm{dBm}$ \\
\hline RX sensitivity & $-87 \mathrm{dBm}$ \\
\hline Carrier frequency & $2.4 \mathrm{GHz}$ \\
\hline MAC & Baseline MAC + EP \\
\hline Superframe length & 32 slots \\
\hline RAP1 length & 16 slots \\
\hline EP length & 16 slots \\
\hline MAC buffer size & 48 packets \\
\hline Channel model & CM3A (hospital room) \\
\hline Mobility model & Object placement \\
\hline \multicolumn{2}{|c|}{ Table 1: System model } \\
\hline $\begin{array}{l}\text { - To implement our system } r \\
\text { Castalia. The extra features } \\
\text { language of OMNeT++. Th } \\
\text { the human subject walks and } \\
\text { The } 9 \text { nodes are placed on bo } \\
\text { and send data packets to the } \\
\text { through a two-hop route to } r \\
\text { number of transmitted and re }\end{array}$ & $\begin{array}{l}\text { model, we modified and extended } \\
\text { can be configured using the NED } \\
\text { he simulation runs for } 60 \mathrm{~s} \text {, while } \\
\text { id places objects in a small room. } \\
\text { pody surface according to Figure } 3 \\
\text { he hub. Some packets may travel } \\
\text { reach the hub. Our metric is the } \\
\text { received packets. }\end{array}$ \\
\hline
\end{tabular}

\section{Channel model}

The quality of a signal in the area of the human body is subject to the typical causes of degradation: fading path loss and shadowing. Path loss can be modeled as a function of distance between two nodes. The model for the communication of the devices placed on body surface for $2.4 \mathrm{GHz}$ according to IEEE 802.15 Working Group is

$$
P L(d)_{\mathrm{dB}}=a \log _{10} d+b+X\left(\mu, \sigma^{2}\right),
$$

where $a=6.6$ and $b=36.1$ are coefficients of linear fitting and $d$ is the distance between two nodes in $\mathrm{mm} . X$ is a normally distributed variable with $\mu=0$ and $\sigma=3.8$.

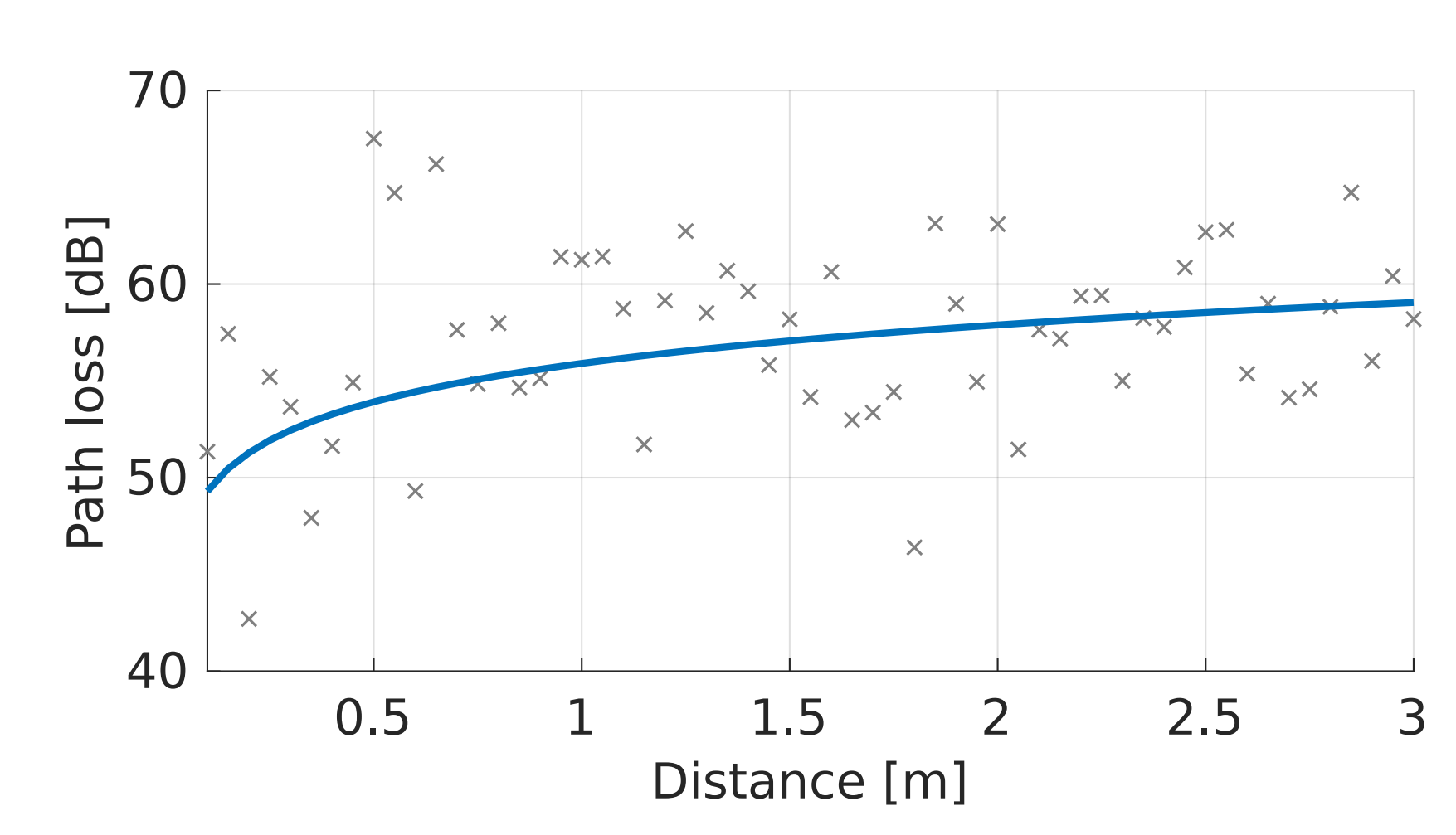

Figure 4: Channel model for $2.4 \mathrm{GHz}$ (CM3A)

- This channel model indicates that we must expect high values for path loss even for distances less than $1 \mathrm{~m}$.
- Nodes placed on human body surface are often disconnected from the network due to very weak signals and high mobility

- We propose a low-level store and forward mechanism in the two-hop area of the body as an enhancement of the standard

- The results show that it is feasible to reach more nodes and receive more data packets

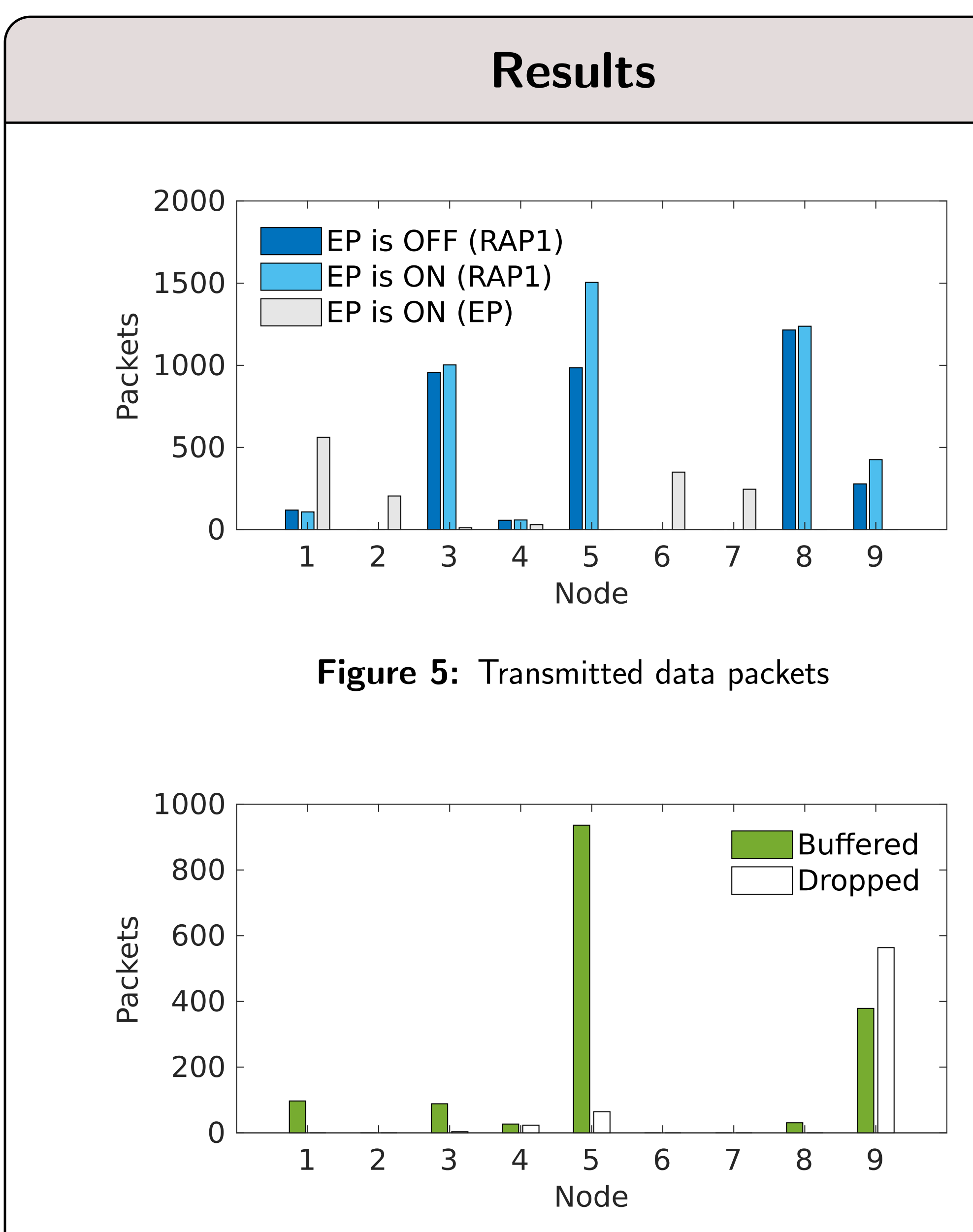

Figure 6: Buffered and Dropped data packets during EP

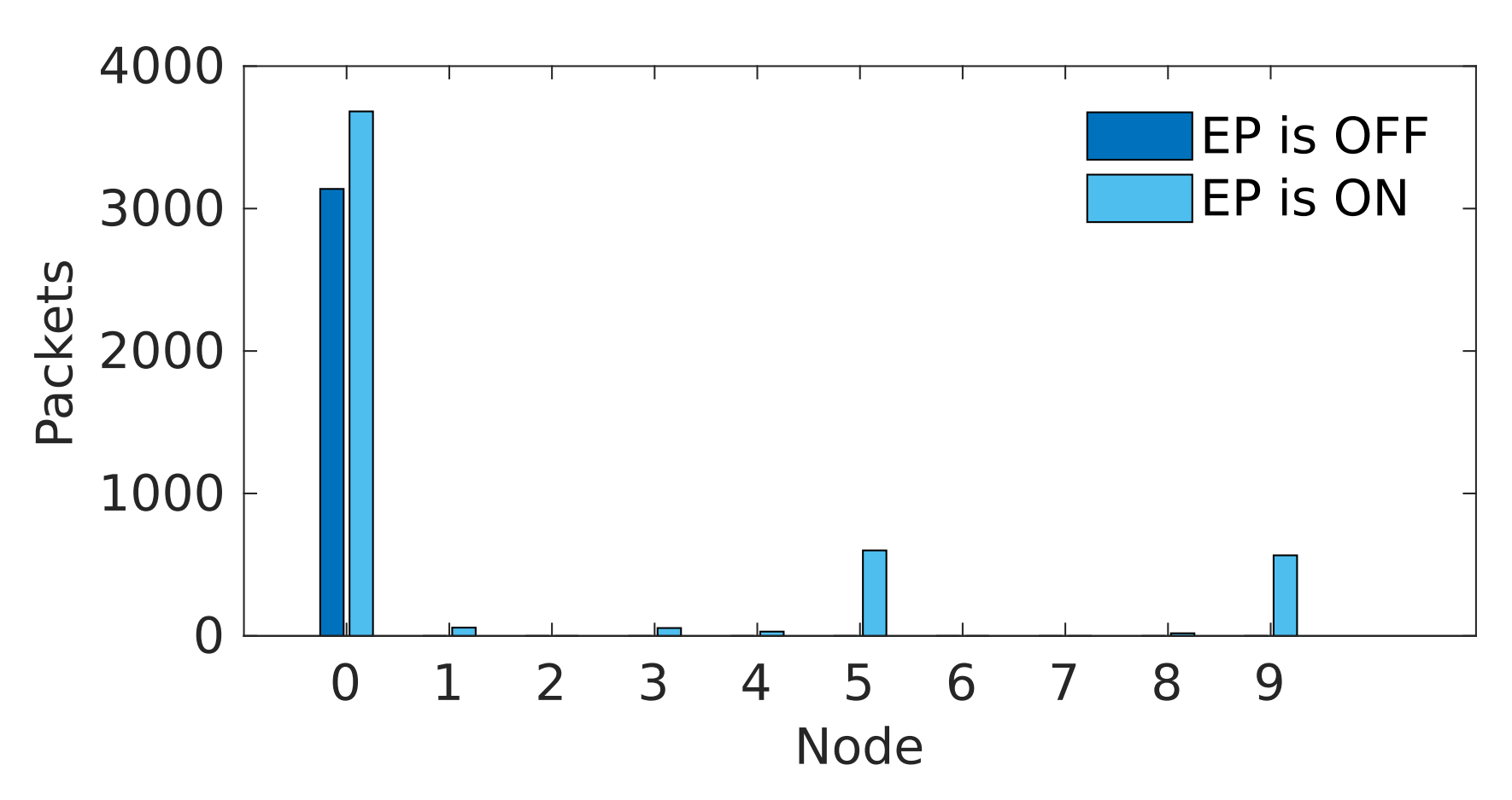

Figure 7: Received data packets

- The results show that when EP is enabled it is feasible to reach distressed nodes and receive more data packets. However, the delivery of each packet to the hub depends on the condition of the corresponding connected node.

\section{Conclusion and Work in Progress}

The concept of relaying works well for BANs and seems to be necessary for the devices with the current sensitivity and low signal levels. The effectiveness of the proposed feature must be validated for more channel and mobility models in order to reach a conclusion. Despite the fact that this work is oriented towards QoS, the quantification of the energy benefit is planned for future work as well.

\section{$\bowtie$ Contact}

Aristotle University of Thessaloniki

Faculty of Engineering

School of Electrical and Computer Engineering 54124, Thessaloniki, Greece

$\{$ cmich, iloridou, niovi\} @auth.gr 